\title{
Extracting Concealed Atrial Premature Contractions from Ambulatory Holter Data Using a PC-Based System
}

\author{
S Mahapatra ${ }^{1}$, B Lin ${ }^{2}$, PJ Wang ${ }^{3}$, NAM Estes III $^{2}$, \\ MK Hammoud ${ }^{2}$, MS Link ${ }^{2}$, A Al-Ahmad ${ }^{3}$ \\ ${ }^{1}$ Mayo Clinic, Rochester, MN, USA \\ ${ }^{2}$ New England Cardiac Arrhythmia Center, Tufts-New England Medical Center, Boston, MA USA \\ ${ }^{3}$ Cardiac Arrhythmia Service, Stanford University, Stanford, CA USA
}

\begin{abstract}
Atrial fibrillation may be cured by eliminating triggering atrial premature contractions (APCs.) Characterizing these APCs may help localize triggers, however this is difficult when the APC is obscured by the preceding $T$-wave. Therefore we sought to develop an inexpensive method to extract APCs from ambulatory Holter monitors in AF patients.

We examined 24-hour Holter strips from patients with AF triggered by APCs. Triggering and nontriggering APCs were extracted using a PC-based T-wave subtraction algorithm. The extracted APCs were compared visually as well as by correlation software.

:The software accurately identified 29 episodes of atrial fibrillation and extracted 76 APCs in 12 patients. In all cases, visual inspection confirmed the presence of atrial fibrillation of APCs. Triggering APCs were similar to other triggering APCs in each patient while nontriggering APCs were less similar to other non-triggering APCs in each patient. Triggering APCs were different from non-triggering APCs.

Automated computerized subtraction of T-waves using an inexpensive $P C$-based system may permit characterization of APCs during ambulatory monitoring.
\end{abstract}

\section{Introduction}

Paroxysmal atrial fibrillation (PAF) maybe initiated by atrial premature contractions (APCs)[1]. Radiofrequency ablation of the source of the triggering APC has cured PAF in some patients. Since an APC's morphology and timing help localize its source, a non-invasive technique to determine the morphology of APCs may be valuable in ablative therapies for PAF [3-6]. However, many APCs have a short coupling interval and may be obscured by the previous QRST complex.

Thus, we sought to build an inexpensive $\mathrm{T}$-wave subtraction system to extract APCs from standard 3-lead ambulatory Holter monitors. We then compared the morphology of triggering and non-triggering APCs..

\section{Methods}

We included patients who had at least 2 episodes of PAF during a 24-hour 3-lead ambulatory Holter monitoring (Rozinn, Glendeale, NY.) Patients who were continuously in atrial fibrillation were excluded.

The Holter recordings were converted to a 16-bit binary format for analysis on a PC (IBM ThinkPad T30, Armonk NY) with a Pentium chip running at $1.8 \mathrm{GHz}$, 512MB and a 40GB harddrive. Next a QRS identification process adapted from Physionet (http://www.physionet.org/physiotools/ecgpuwave) was used. This method has been previously described [2]. Briefly, the strip was sequentially passed through a low and high-pass filters. Next the first derivative of the result was taken and then this derivative was squared and window interloped. This technique identifies QRS complexes with over 95\% sensitivity and specificity (figure 1.)

After QRS identification, the R-to-R intervals were calculated. Intervals with beat-to-beat variability of greater than 15 percent were labeled atrial fibrillation. This diagnosis was confirmed by visual inspection.

All QRST complexes within 30 minutes of an episode of atrial fibrillation were subtracted from the average of the five previous QRST complexes. If subtraction left a signal greater than $1.5 \mathrm{mV}$ in height and $60 \mathrm{~ms}$ in length it was marked as a possible APC. It was then confirmed visually. APCs less than 10 seconds prior to PAF were labeled a "triggering APCs" while others were called "non-triggering APCs." This technique was repeated for each lead (figure 2).

Each APC was compared visually and by correlation software to other triggering APCs and non-triggering APCs in the same individual

APCs were visually compared by three investigators who were blinded to the patients' clinical data as well as 


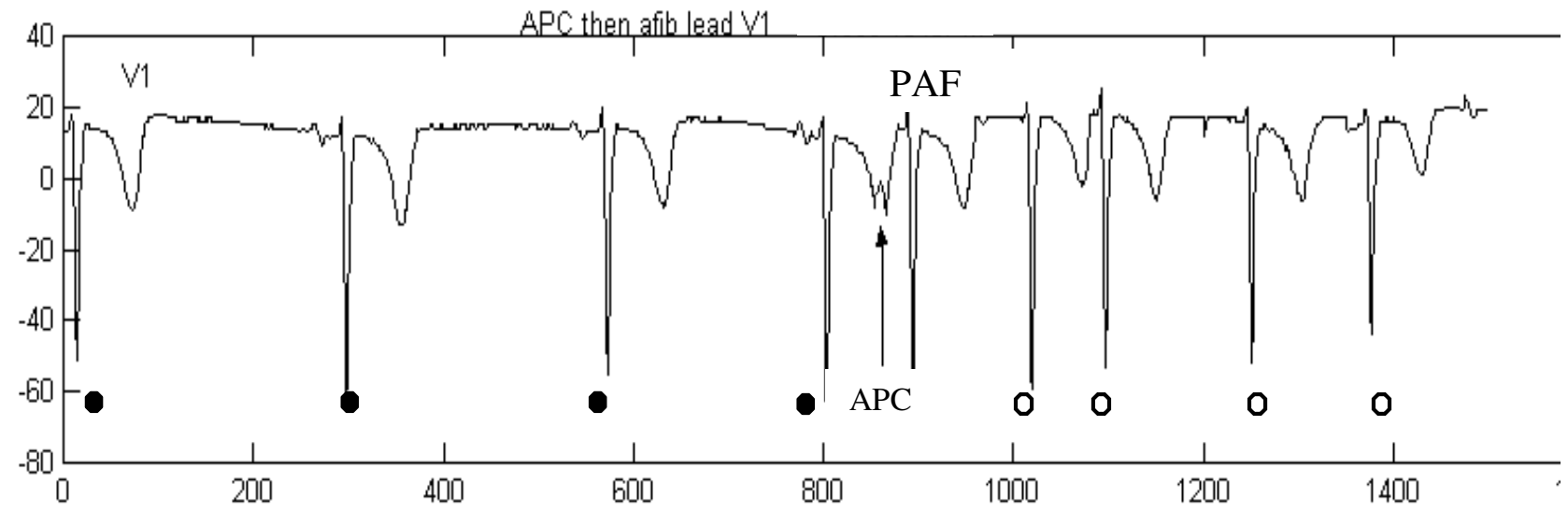

Figure 1: QRS complexes marked (dots) and periods with irregular intervals are marked as PAF (open dots)

Note an APC that starts PAF. This APC is revealed by T-wave subtraction in Figure 2.

to its triggering or non-triggering status.

Correlation waveform analysis was performed using Matlab (version 5.1 Natick, MA) software yielding a correlation coefficient.

Each lead was compared independently. To characterize the timing of triggering and non-triggering APCs, the prematurity index (PMI) of each APC was calculated. The PMI of is equal to the time from the last normal sinus $\mathrm{P}$ wave to the start of the APC divided by the underlying sinus cycle length. The PMI of each APC was compared to other PMIs in a given patient..

\section{Results}

Twelve patients were included (4 women, mean age $55+/-11$ years.) Analysis took $702 \pm 11$ seconds per lead for 24-hours of data. The software identified 29 episodes of PAF all preceded by triggering APCs. All episodes were confirmed visually. The mean cycle length of these episodes was $500 \pm 72 \mathrm{~ms}$ and the mean duration was $48 \pm 12$ minutes.

Software identified a total of 76 APCs (29 triggering, 47 non-triggering.) in all 3 leads. Since each APC had 3 leads (V1, V5, III) there were 228 APC waveforms to compare (87 triggering, 141 non-triggering.) All APCs were confirmed by visual inspection.

Visual comparison showed 81 of the 87 triggering APC comparisons were matched (0.93 correlation.) while software correlation calculated a mean correlation coefficient of 0.80 . Visual comparison showed 99 of the 141 non-triggering APC comparisons were matched $(0.72$ correlation.) while software correlation calculated a mean correlation coefficient of 0.64. When comparing triggering to non-triggering APCs, visual comparison showed that 22 of the 81 triggering APCs matched at least one of the patient's non-triggering APCs $(0.28$ correlation while software correlation calculated a mean correlation coefficient of 0.11

Seven patients had a single triggering APC morphology that differed from all their non-triggering APCs. Three patients had 2 different triggering APCs that both differed from the non-triggering APCs. One patient had only one type of APC and one had multiple types of APCs none which matched each other.

The PMI of triggering APCs was not different from the PMI of non-triggering APCs (0.81 vs $0.75, \mathrm{p}=\mathrm{ns})$

The total system cost was $\$ 3211$ when purchased new22 of the 81 triggering APCs matched at least one of the patient's non-triggering APCs $(0.28$ correlation while software correlation calculated a mean correlation coefficient of 0.11 .

Seven patients had a single triggering APC

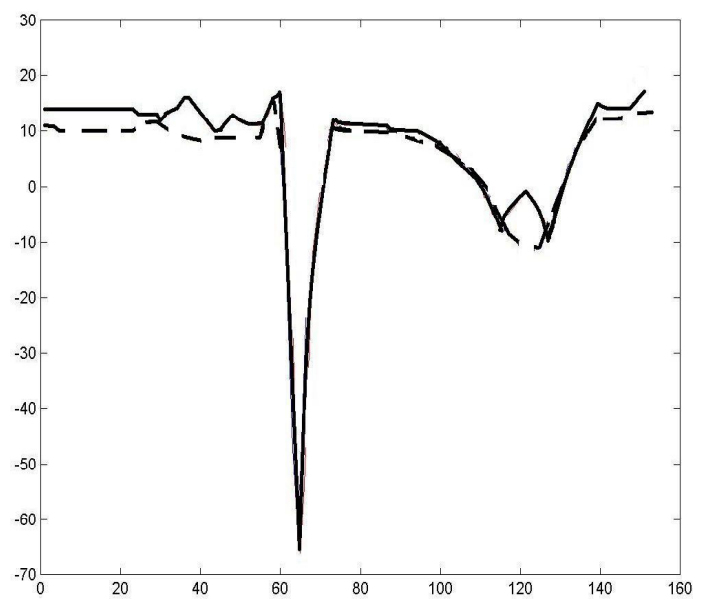

Figure 2: Each QRST complex (solid line) is subtracted from the average of the previous five QRST complexes (dotted line) in a search for APCs. 
morphology that differed from all their non-triggering APCs. Three patients had 2 different triggering APCs that both differed from the non-triggering APCs. One patient had only one type of APC and one had multiple types of APCs none which matched each other.

The PMI of triggering APCs was not different from the PMI of non-triggering APCs (0.81 vs $0.75, \mathrm{p}=\mathrm{ns}$ )

The total system cost was $\$ 3211$ when purchased new.

\section{Discussion and conclusions}

Our study introduces an inexpensive, PC-based system that can identify and extract APCs from ambulatory Holter data in patients with PAF.

Previous investigators have shown that the morphology of an APC correlates with its site of origin. In 1910, Sir Thomas Lewis showed that pacing different parts of the canine atrium resulted in distinct $P$ wave morphologies [7]. Later studies have shown that certain $\mathrm{P}$ wave morphologies suggest particular sites of origin [814]. SippensGroenewegen et al went further by creating an atlas of $\mathrm{P}$ wave integral maps to localize the site of origin of right atrial arrhythmias[15]. More recently, Choi et al showed that the APC morphology correlated with its location on invasive testing. These results confirm that APC morphology predicts APC location[16].

Timing of the $\mathrm{P}$ wave has also shown to be important. In reference to PAF ablation, Haissaguerre et al showed that $\mathrm{P}$ on $\mathrm{T}$ extrasystole is highly predicative of a pulmonary vein focus [3].

Previous investigators have used T-wave subtraction to reveal APCs buried in the repolarization sequence. However, manual techniques can be time consuming and inexact, while specialized, commercially developed systems can be prohibitively expensive.

By using a PCs and commercial software, we were able to create an inexpensive digital method for identifying PAF, extracting APCs, and classifying the APCs as triggering or non-triggering. All episodes of PAF and the extracted APCs were confirmed by visual inspection.

Furthermore using this system, we have shown that in most patients each triggering APC is similar to other triggering APC but different from non-triggering APCs. This is in agreement with our visual inspection and suggests that triggering APCs arise from a single focus that could be a target for ablation or isolation.

We have developed an inexpensive, tool to extract and characterize triggering APCs and differentiate them from non-triggering APCs. This tool may aide in selecting PAF patients would benefit from ablation and guide ablation.

\section{References}

[1] Haissaguerre $M$, et al. Spontaneous initiation of atrial fibrillation by ectopic beats originating in the pulmonary veins. N Engl J Med 1998;339:659-66.

[2] Pan J, Tompkins WJ. A Real Time QRS Detection Algorithm IEEE Transactions on Biomedical Engineering; 1984 BME-32:230-236

[3] Haissaguerre $\mathrm{M}$, et al. $\mathrm{P}$ on $\mathrm{T}$ extrasystoles are highly predictive of pulmonary vein origin. PACE Abstracts 1999;22:836.

[4] Throne RD, et al. Discrimination of retrograde from anterograde atrial activation using intracardiac electrogram waveform analysis. PACE 1989;12:1622-30.

[5] Fitzgerald DM, et al. P wave morphology during atrial pacing along the atrioventricular ring. ECG localization of the site of origin of retrograde atrial activation. Journal of Electrocardiology 1996;29:1-10.

[6] Kuchar DL, Kelly R Thorburn CW. High-frequency analysis of the surface electrocardiograms of patients with supraventricular tachycardia: accurate identification of atrial activation and determination of the mechanism of tachycardia. Circulation 1986;74:1016-26.

[7] Lewis T. Galvanometric curves yielded by cardiac beats generated in various areas of the auricular musculature. Heart 1910;2:68-76.

[8] Tang CW, et al, Use of $\mathrm{P}$ wave configuration during atrial tachycardia to predict site of origin. Journal of the American College of Cardiology 1995;26:1315-24.

[9] Beder S.D, et al., Clinical confirmation of ECG criteria for left atrial rhythm. American Heart Journal 1982;103:84852.

[10] Somlyo A, Garyzel J. Left atrial arrhythmias. Amercan Heart Journal 1977;65:68-76.

[11] Gillette PC, Garson A. Electrophysiologic and pharmacologic characteristics of automatic ectopic atrial tachycardia. Circulation 1977;56:571-5.

[12] Mirowski, M., et al. Negative $P$ waves in lead I detrversion: Differential diagnosis from mirror-image detrocardia. Circulation 1962;26:413.

[13] Gelb BD, Garson A. Noninvasive discrimination of right atrial ectopic tachycardia from sinus tachycardia in "dilated cardiomyopathy". American Heart Journal 1990;120:88691.

[14] SippensGroenewegen A, et al. Body surface mapping of atrial arrhythmias: atlas of paced $\mathrm{P}$ wave integral maps to localize the focal origin of right atrial tachycardia. Journal of Electrocardiology 1998;31:85-91.

[15] Choi KJ, et al. QRST subtraction combined with a pacemap catalogue for the prediction of ectopy source by surface electrocardiogram in patients with paroxysmal atrial fibrillation. J Am Coll Cardiol 2002;40:2013-21.

Address for Correspondence:

Srijoy Mahapatra, MD

200 First Street, SW

Rochester, MN 55901

mahapatra.srijoy@mayo.edu 